\title{
Observation of Electron Neutrino Appearance in a Muon Neutrino Beam
}

K. Abe ${ }^{46}$ J. Adam,${ }^{32}$ H. Aihara, ${ }^{45,23}$ T. Akiri, ${ }^{9}$ C. Andreopoulos, ${ }^{44}$ S. Aoki, ${ }^{24}$ A. Ariga, ${ }^{2}$ T. Ariga,${ }^{2}$ S. Assylbekov, ${ }^{8}$ D. Autiero, ${ }^{29}$ M. Barbi, ${ }^{39}$ G. J. Barker,${ }^{54}$ G. Barr, ${ }^{35}$ M. Bass, ${ }^{8}$ M. Batkiewicz,${ }^{13}$ F. Bay, ${ }^{11}$ S. W. Bentham, ${ }^{26}$ V. Berardi, ${ }^{18}$ B. E. Berger, ${ }^{8}$ S. Berkman, ${ }^{4}$ I. Bertram,${ }^{26}$ S. Bhadra ${ }^{58}$ F. d. M. Blaszczyk, ${ }^{28}$ A. Blondel, ${ }^{12}$ C. Bojechko, ${ }^{51}$ S. Bordoni ${ }^{15}$ S. B. Boyd,${ }^{54}$ D. Brailsford, ${ }^{17}$ A. Bravar, ${ }^{12}$ C. Bronner, ${ }^{25}$ N. Buchanan, ${ }^{8}$ R. G. Calland, ${ }^{27}$ J. Caravaca Rodríguez, ${ }^{15}$ S. L. Cartwright, ${ }^{42}$ R. Castillo, ${ }^{15}$ M. G. Catanesi, ${ }^{18}$ A. Cervera, ${ }^{16}$ D. Cherdack, ${ }^{8}$ G. Christodoulou, ${ }^{27}$ A. Clifton, ${ }^{8}$ J. Coleman, ${ }^{27}$ S. J. Coleman, ${ }^{7}$ G. Collazuol, ${ }^{20}$ K. Connolly, ${ }^{55}$ L. Cremonesi, ${ }^{38}$ A. Dabrowska, ${ }^{13}$ I. Danko, ${ }^{37}$ R. Das, ${ }^{8}$ S. Davis, ${ }^{55}$ P. de Perio, ${ }^{49}$ G. De Rosa, ${ }^{19}$ T. Dealtry, ${ }^{44,35}$ S. R. Dennis,${ }^{54,44}$ C. Densham, ${ }^{44}$ F. Di Lodovico, ${ }^{38}$ S. Di Luise, ${ }^{11}$ O. Drapier, ${ }^{10}$ T. Duboyski, ${ }^{38}$ K. Duffy, ${ }^{35}$ F. Dufour, ${ }^{12}$ J. Dumarchez,${ }^{36}$ S. Dytman ${ }^{37}$ M. Dziewiecki, ${ }^{53}$ S. Emery, ${ }^{6}$ A. Ereditato, ${ }^{2}$ L. Escudero, ${ }^{16}$ A. J. Finch, ${ }^{26}$ L. Floetotto, ${ }^{41}$ M. Friend ${ }^{14, \dagger}$ Y. Fujii, ${ }^{14, \dagger}$ Y. Fukuda, ${ }^{30}$ A. P. Furmanski, ${ }^{54}$ V. Galymov, ${ }^{6}$ A. Gaudin, ${ }^{51}$ S. Giffin,${ }^{39}$ C. Giganti, ${ }^{36}$ K. Gilje, ${ }^{32}$ D. Goeldi, ${ }^{2}$ T. Golan,${ }^{57}$ J. J. Gomez-Cadenas, ${ }^{16}$ M. Gonin,${ }^{10}$ N. Grant ${ }^{26}$ D. Gudin, ${ }^{22}$ D. R. Hadley, ${ }^{54}$ A. Haesler, ${ }^{12}$ M. D. Haigh,${ }^{54}$ P. Hamilton, ${ }^{17}$ D. Hansen,${ }^{37}$ T. Hara ${ }^{24}$ M. Hartz,${ }^{23,50}$ T. Hasegawa, ${ }^{14, \dagger}$ N. C. Hastings, ${ }^{39}$ Y. Hayato, ${ }^{46}$ C. Hearty, ${ }^{4+}$ R. L. Helmer, ${ }^{50}$ M. Hierholzer, ${ }^{2}$ J. Hignight, ${ }^{32}$ A. Hillairet,,${ }^{51}$ A. Himmel, ${ }^{9}$ T. Hiraki, ${ }^{25}$ S. Hirota, ${ }^{25}$ J. Holeczek,${ }^{43}$ S. Horikawa, ${ }^{11}$ K. Huang, ${ }^{25}$ A. K. Ichikawa, ${ }^{25}$ K. Ieki, ${ }^{25}$ M. Ieva, ${ }^{15}$ M. Ikeda, ${ }^{46}$ J. Imber, ${ }^{32}$ J. Insler, ${ }^{28}$ T. J. Irvine, ${ }^{47}$ T. Ishida, ${ }^{14, \dagger}$ T. Ishii, ${ }^{14, \dagger}$ S. J. Ives, ${ }^{17}$ K. Iyogi ${ }^{46}$ A. Izmaylov, ${ }^{16,22}$ A. Jacob ${ }^{35}$ B. Jamieson, ${ }^{56}$ R. A. Johnson, ${ }^{7}$ J. H. Jo, ${ }^{32}$ P. Jonsson, ${ }^{17}$ C. K. Jung, ${ }^{32,8}$ A. C. Kaboth, ${ }^{17}$ T. Kajita, ${ }^{47,}$ H. Kakuno, ${ }^{48}$ J. Kameda ${ }^{46}$ Y. Kanazawa, ${ }^{45}$ D. Karlen, ${ }^{51,50}$ I. Karpikov, ${ }^{22}$ E. Kearns, ${ }^{3,23,8}$ M. Khabibullin, ${ }^{22}$ A. Khotjantsev, ${ }^{22}$ D. Kielczewska, ${ }^{52}$ T. Kikawa, ${ }^{25}$ A. Kilinski, ${ }^{31}$ J. Kim, ${ }^{4}$ J. Kisiel, ${ }^{43}$ P. Kitching, ${ }^{1}$ T. Kobayashi, ${ }^{14, \dagger}$ L. Koch,${ }^{41}$ A. Kolaceke, ${ }^{39}$ A. Konaka, ${ }^{50}$ L. L. Kormos, ${ }^{26}$ A. Korzenev,${ }^{12}$ K. Koseki, ${ }^{14, \dagger}$ Y. Koshio,${ }^{33,8}$ I. Kreslo, ${ }^{2}$ W. Kropp, ${ }^{5}$ H. Kubo, ${ }^{25}$ Y. Kudenko, ${ }^{22, \|}$ S. Kumaratunga, ${ }^{50}$ R. Kurjata, ${ }^{53}$ T. Kutter, ${ }^{28}$ J. Lagoda,${ }^{31}$ K. Laihem, ${ }^{41}$ I. Lamont, ${ }^{26}$ M. Laveder, ${ }^{20}$ M. Lawe, ${ }^{42}$ M. Lazos, ${ }^{27}$ K. P. Lee, ${ }^{47}$ C. Licciardi, ${ }^{39}$ T. Lindner, ${ }^{50}$ C. Lister, ${ }^{54}$ R. P. Litchfield ${ }^{54}$ A. Longhin,${ }^{20}$ L. Ludovici, ${ }^{21}$ M. Macaire, ${ }^{6}$ L. Magaletti, ${ }^{18}$ K. Mahn,${ }^{50}$ M. Malek, ${ }^{17}$ S. Manly, ${ }^{40}$ A. D. Marino, ${ }^{7}$ J. Marteau, ${ }^{29}$ J. F. Martin, ${ }^{49}$ T. Maruyama, ${ }^{14, \dagger}$ J. Marzec, ${ }^{53}$ E. L. Mathie, ${ }^{39}$ V. Matveev, ${ }^{22}$ K. Mavrokoridis, ${ }^{27}$ E. Mazzucato, ${ }^{6}$ M. McCarthy, ${ }^{4}$ N. McCauley, ${ }^{27}$ K. S. McFarland, ${ }^{40}$ C. McGrew, ${ }^{32}$ C. Metelko, ${ }^{27}$ M. Mezzetto, ${ }^{20}$ P. Mijakowski, ${ }^{31}$ C. A. Miller, ${ }^{50}$ A. Minamino, ${ }^{25}$ O. Mineev, ${ }^{22}$ S. Mine, ${ }^{5}$ A. Missert, ${ }^{7}$ M. Miura, ${ }^{46,}$ L. Monfregola, ${ }^{16}$ S. Moriyama, ${ }^{46,8}$ Th. A. Mueller, ${ }^{10}$ A. Murakami, ${ }^{25}$ M. Murdoch ${ }^{27}$ S. Murphy, ${ }^{11}$ J. Myslik, ${ }^{51}$ T. Nagasaki,${ }^{25}$ T. Nakadaira,${ }^{14, \dagger}$ M. Nakahata, ${ }^{46,23}$ T. Nakai,${ }^{34}$ K. Nakamura, ${ }^{23,14, \dagger}$

S. Nakayama, ${ }^{46,8}$ T. Nakaya,${ }^{25,23}$ K. Nakayoshi, ${ }^{14, \dagger}$ D. Naples,${ }^{37}$ C. Nielsen, ${ }^{4}$ M. Nirkko, ${ }^{2}$ K. Nishikawa, ${ }^{14, \dagger}$ Y. Nishimura, ${ }^{47}$ H. M. O’Keeffe, ${ }^{26}$ R. Ohta, ${ }^{14, \dagger}$ K. Okumura, ${ }^{47,23}$ T. Okusawa, ${ }^{34}$ W. Oryszczak, ${ }^{52}$ S. M. Oser, ${ }^{4}$ R. A. Owen, ${ }^{38}$ Y. Oyama, ${ }^{14, \dagger}$ V. Palladino, ${ }^{19}$ V. Paolone,${ }^{37}$ D. Payne,${ }^{27}$ G. F. Pearce ${ }^{44}$ O. Perevozchikov, ${ }^{28}$ J. D. Perkin,${ }^{42}$ Y. Petrov, ${ }^{4}$ L. J. Pickard, ${ }^{42}$ E. S. Pinzon Guerra, ${ }^{58}$ C. Pistillo, ${ }^{2}$ P. Plonski,${ }^{53}$ E. Poplawska, ${ }^{38}$ B. Popov, ${ }^{36,}$ M. Posiadala, ${ }^{52}$ J.-M. Poutissou, ${ }^{50}$ R. Poutissou, ${ }^{50}$ P. Przewlocki, ${ }^{31}$ B. Quilain, ${ }^{10}$ E. Radicioni, ${ }^{18}$ P. N. Ratoff, ${ }^{26}$ M. Ravonel, ${ }^{12}$ M. A. M. Rayner, ${ }^{12}$ A. Redij, ${ }^{2}$ M. Reeves, ${ }^{26}$ E. Reinherz-Aronis, ${ }^{8}$ F. Retiere ${ }^{50}$ A. Robert, ${ }^{36}$ P. A. Rodrigues, ${ }^{40}$ P. Rojas, ${ }^{8}$ E. Rondio, ${ }^{31}$ S. Roth, ${ }^{41}$ A. Rubbia, ${ }^{11}$

D. Ruterbories, ${ }^{8}$ R. Sacco, ${ }^{38}$ K. Sakashita, ${ }^{14, \dagger}$ F. Sánchez, ${ }^{15}$ F. Sato, ${ }^{14}$ E. Scantamburlo, ${ }^{12}$ K. Scholberg,,${ }^{9}$ J. Schwehr, ${ }^{8}$ M. Scott,${ }^{50}$ Y. Seiya ${ }^{34}$ T. Sekiguchi, ${ }^{14, \dagger}$ H. Sekiya, ${ }^{46,8}$ D. Sgalaberna, ${ }^{11}$ M. Shiozawa, ${ }^{46,23}$ S. Short, ${ }^{17}$ Y. Shustrov, ${ }^{22}$ P. Sinclair, ${ }^{17}$ B. Smith, ${ }^{17}$ R. J. Smith, ${ }^{35}$ M. Smy, J. T. Sobczyk, ${ }^{57}$ H. Sobel, ${ }^{5,23}$ M. Sorel, ${ }^{16}$ L. Southwell, ${ }^{26}$ P. Stamoulis, ${ }^{16}$ J. Steinmann, ${ }^{41}$ B. Still, ${ }^{38}$ Y. Suda, ${ }^{45}$ A. Suzuki, ${ }^{24}$ K. Suzuki, ${ }^{25}$ S. Y. Suzuki, ${ }^{14 \dagger}$ Y. Suzuki, ${ }^{46,23}$ T. Szeglowski, ${ }^{43}$

R. Tacik,${ }^{39,50}$ M. Tada, ${ }^{14, \dagger}$ S. Takahashi, ${ }^{25}$ A. Takeda, ${ }^{46}$ Y. Takeuchi, ${ }^{24,23}$ H. K. Tanaka, ${ }^{46,8}$ H. A. Tanaka, ${ }^{4, \$}$ M. M. Tanaka, ${ }^{14, \dagger}$ D. Terhorst, ${ }^{41}$ R. Terri, ${ }^{38}$ L. F. Thompson, ${ }^{42}$ A. Thorley, ${ }^{27}$ S. Tobayama, ${ }^{4}$ W. Toki, ${ }^{8}$ T. Tomura, ${ }^{46}$ Y. Totsuka, C. Touramanis, ${ }^{27}$ T. Tsukamoto, ${ }^{14, \dagger}$ M. Tzanov, ${ }^{28}$ Y. Uchida, ${ }^{17}$ K. Ueno, ${ }^{46}$ A. Vacheret ${ }^{35}$ M. Vagins,${ }^{23,5}$ G. Vasseur,${ }^{6}$ T. Wachala, ${ }^{13}$ A. V. Waldron,${ }^{35}$ C. W. Walter, ${ }^{9,8}$ D. Wark,${ }^{44,17}$ M. O. Wascko, ${ }^{17}$ A. Weber, ${ }^{44,35}$ R. Wendell, ${ }^{46,8}$ R. J. Wilkes ${ }^{55}$ M. J. Wilking, ${ }^{50}$ C. Wilkinson, ${ }^{42}$ Z. Williamson,${ }^{35}$ J. R. Wilson, ${ }^{38}$ R. J. Wilson, ${ }^{8}$ T. Wongjirad, ${ }^{9}$ Y. Yamada, ${ }^{14, \dagger}$

K. Yamamoto, ${ }^{34}$ C. Yanagisawa, ${ }^{32, * *}$ S. Yen, ${ }^{50}$ N. Yershov, ${ }^{22}$ M. Yokoyama, ${ }^{45,}$ T. Yuan, ${ }^{7}$ A. Zalewska, ${ }^{13}$ J. Zalipska, ${ }^{31}$ L. Zambelli, ${ }^{36}$ K. Zaremba, ${ }^{53}$ M. Ziembicki, ${ }^{53}$ E. D. Zimmerman, ${ }^{7}$ M. Zito, ${ }^{6}$ J. Żmuda ${ }^{57}$

\section{(T2K Collaboration)}

\footnotetext{
${ }^{1}$ University of Alberta, Centre for Particle Physics, Department of Physics, Edmonton, Alberta, Canada

${ }^{2}$ University of Bern, Albert Einstein Center for Fundamental Physics, Laboratory for High Energy Physics (LHEP), Bern, Switzerland ${ }^{3}$ Boston University, Department of Physics, Boston, Massachusetts, USA

${ }^{4}$ University of British Columbia, Department of Physics and Astronomy, Vancouver, British Columbia, Canada
} 
${ }^{5}$ University of California, Irvine, Department of Physics and Astronomy, Irvine, California, USA

${ }^{6}$ IRFU, CEA Saclay, Gif-sur-Yvette, France

${ }^{7}$ University of Colorado at Boulder, Department of Physics, Boulder, Colorado, USA

${ }^{8}$ Colorado State University, Department of Physics, Fort Collins, Colorado, USA

${ }^{9}$ Duke University, Department of Physics, Durham, North Carolina, USA

${ }^{10}$ Ecole Polytechnique, IN2P3-CNRS, Laboratoire Leprince-Ringuet, Palaiseau, France

${ }^{11}$ ETH Zurich, Institute for Particle Physics, Zurich, Switzerland

${ }^{12}$ University of Geneva, Section de Physique, DPNC, Geneva, Switzerland

${ }^{13}$ H. Niewodniczanski Institute of Nuclear Physics PAN, Cracow, Poland

${ }^{14}$ High Energy Accelerator Research Organization (KEK), Tsukuba, Ibaraki, Japan

${ }^{15}$ Institut de Fisica d'Altes Energies (IFAE), Bellaterra (Barcelona), Spain

${ }^{16}$ IFIC (CSIC and University of Valencia), Valencia, Spain

${ }^{17}$ Imperial College London, Department of Physics, London, United Kingdom

${ }^{18}$ INFN Sezione di Bari and Università e Politecnico di Bari, Dipartimento Interuniversitario di Fisica, Bari, Italy

${ }^{19}$ INFN Sezione di Napoli and Università di Napoli, Dipartimento di Fisica, Napoli, Italy

${ }^{20}$ INFN Sezione di Padova and Università di Padova, Dipartimento di Fisica, Padova, Italy

${ }^{21}$ INFN Sezione di Roma and Università di Roma "La Sapienza”, Roma, Italy

${ }^{22}$ Institute for Nuclear Research of the Russian Academy of Sciences, Moscow, Russia

${ }^{23}$ Kavli Institute for the Physics and Mathematics of the Universe (WPI), Todai Institutes for Advanced Study, University of Tokyo, Kashiwa, Chiba, Japan

${ }^{24}$ Kobe University, Kobe, Japan

${ }^{25}$ Kyoto University, Department of Physics, Kyoto, Japan

${ }^{26}$ Lancaster University, Physics Department, Lancaster, United Kingdom

${ }^{27}$ University of Liverpool, Department of Physics, Liverpool, United Kingdom

${ }^{28}$ Louisiana State University, Department of Physics and Astronomy, Baton Rouge, Louisiana, USA

${ }^{29}$ Université de Lyon, Université Claude Bernard Lyon 1, IPN Lyon (IN2P3), Villeurbanne, France

${ }^{30}$ Miyagi University of Education, Department of Physics, Sendai, Japan

${ }^{31}$ National Centre for Nuclear Research, Warsaw, Poland

${ }^{32}$ State University of New York at Stony Brook, Department of Physics and Astronomy, Stony Brook, New York, USA

${ }^{33}$ Okayama University, Department of Physics, Okayama, Japan

${ }^{34}$ Osaka City University, Department of Physics, Osaka, Japan

${ }^{35}$ Oxford University, Department of Physics, Oxford, United Kingdom

${ }^{36}$ UPMC, Université Paris Diderot, CNRS/IN2P3, Laboratoire de Physique Nucléaire et de Hautes Energies (LPNHE), Paris, France

${ }^{37}$ University of Pittsburgh, Department of Physics and Astronomy, Pittsburgh, Pennsylvania, USA

${ }^{38}$ Queen Mary University of London, School of Physics and Astronomy, London, United Kingdom

${ }^{39}$ University of Regina, Department of Physics, Regina, Saskatchewan, Canada

${ }^{40}$ University of Rochester, Department of Physics and Astronomy, Rochester, New York, USA

${ }^{41}$ RWTH Aachen University, III. Physikalisches Institut, Aachen, Germany

${ }^{42}$ University of Sheffield, Department of Physics and Astronomy, Sheffield, United Kingdom

${ }^{43}$ University of Silesia, Institute of Physics, Katowice, Poland

${ }^{44}$ STFC, Rutherford Appleton Laboratory, Harwell Oxford, and Daresbury Laboratory, Warrington, United Kingdom

${ }^{45}$ University of Tokyo, Department of Physics, Tokyo, Japan

${ }^{46}$ University of Tokyo, Institute for Cosmic Ray Research, Kamioka Observatory, Kamioka, Japan

${ }^{47}$ University of Tokyo, Institute for Cosmic Ray Research, Research Center for Cosmic Neutrinos, Kashiwa, Japan

${ }^{48}$ Tokyo Metropolitan University, Department of Physics, Tokyo, Japan

${ }^{49}$ University of Toronto, Department of Physics, Toronto, Ontario, Canada

${ }^{50}$ TRIUMF, Vancouver, British Columbia, Canada

${ }^{51}$ University of Victoria, Department of Physics and Astronomy, Victoria, British Columbia, Canada

${ }^{52}$ University of Warsaw, Faculty of Physics, Warsaw, Poland

${ }^{53}$ Warsaw University of Technology, Institute of Radioelectronics, Warsaw, Poland

${ }^{54}$ University of Warwick, Department of Physics, Coventry, United Kingdom

${ }^{55}$ University of Washington, Department of Physics, Seattle, Washington, USA

${ }^{56}$ University of Winnipeg, Department of Physics, Winnipeg, Manitoba, Canada

${ }^{57}$ Wroclaw University, Faculty of Physics and Astronomy, Wroclaw, Poland

${ }^{58}$ York University, Department of Physics and Astronomy, Toronto, Ontario, Canada

(Received 19 November 2013; published 10 February 2014)

The T2K experiment has observed electron neutrino appearance in a muon neutrino beam produced $295 \mathrm{~km}$ from the Super-Kamiokande detector with a peak energy of $0.6 \mathrm{GeV}$. A total of 28 electron neutrino events were detected with an energy distribution consistent with an appearance signal, 
corresponding to a significance of $7.3 \sigma$ when compared to $4.92 \pm 0.55$ expected background events. In the Pontecorvo-Maki-Nakagawa-Sakata mixing model, the electron neutrino appearance signal depends on several parameters including three mixing angles $\theta_{12}, \theta_{23}, \theta_{13}$, a mass difference $\Delta m_{32}^{2}$ and a $C P$ violating phase $\delta_{C P}$. In this neutrino oscillation scenario, assuming $\left|\Delta m_{32}^{2}\right|=2.4 \times 10^{-3} \mathrm{eV}^{2}, \sin ^{2} \theta_{23}=0.5$, and $\Delta m_{32}^{2}>0\left(\Delta m_{32}^{2}<0\right)$, a best-fit value of $\sin ^{2} 2 \theta_{13}=0.140_{-0.032}^{+0.038}\left(0.170_{-0.037}^{+0.045}\right)$ is obtained at $\delta_{C P}=0$. When combining the result with the current best knowledge of oscillation parameters including the world average value of $\theta_{13}$ from reactor experiments, some values of $\delta_{C P}$ are disfavored at the $90 \%$ C.L.

Introduction.-The discovery of neutrino oscillations using atmospheric neutrinos was made by SuperKamiokande in 1998 [1]. Since then, many other experiments have confirmed the phenomenon of neutrino oscillations through various disappearance modes of flavor transformations. However, to date, there has not been an observation of the explicit appearance of a different neutrino flavor from neutrinos of another flavor through neutrino oscillations. In 2011, the T2K collaboration published the first indication of electron neutrino appearance from a muon neutrino beam at $2.5 \sigma$ significance based on a data set corresponding to $1.43 \times 10^{20}$ protons on target (POT) [2,3]. This result was followed by the publication of further evidence for electron neutrino appearance at $3.1 \sigma$ in early 2013 [4]. This Letter presents new results from the $\mathrm{T} 2 \mathrm{~K}$ experiment that establish, at greater than $5 \sigma$, the observation of electron-neutrino appearance from a muon-neutrino beam.

In a three-flavor framework, neutrino oscillations are described by the Pontecorvo-Maki-Nakagawa-Sakata (PMNS) matrix $[5,6]$ which is parametrized by three mixing angles $\theta_{12}, \theta_{23}, \theta_{13}$, and a $C P$ violating phase $\delta_{C P}$. In this framework, the probability for $\nu_{\mu} \rightarrow \nu_{e}$ oscillation can be expressed [7] as

$$
\begin{aligned}
& P\left(\nu_{\mu} \rightarrow \nu_{e}\right) \simeq \sin ^{2} \theta_{23} \sin ^{2} 2 \theta_{13} \sin ^{2} \frac{\Delta m_{31}^{2} L}{4 E} \\
& -\frac{\sin 2 \theta_{12} \sin 2 \theta_{23}}{2 \sin \theta_{13}} \sin \frac{\Delta m_{21}^{2} L}{4 E} \sin ^{2} 2 \theta_{13} \sin ^{2} \frac{\Delta m_{31}^{2} L}{4 E} \sin \delta_{C P} \\
& \quad+(C P \text { even term, solar term, matter effectterm })
\end{aligned}
$$

where $L$ is the neutrino propagation distance and $E$ is the neutrino energy. The measurement of $\nu_{\mu} \rightarrow \nu_{e}$ oscillations is of particular interest because this mode is sensitive to both $\theta_{13}$ and $\delta_{C P}$. The first indication of nonzero $\theta_{13}$ was published by T2K [3] based on the measurement of $\nu_{\mu} \rightarrow$ $\nu_{e}$ oscillations. More recently, indications of $\nu_{\mu} \rightarrow \nu_{e}$ oscillations were also reported by the MINOS experiment [8]. The value of $\theta_{13}$ is now precisely known to be $9.1^{\circ} \pm$ $0.6^{\circ}$ from measurements of $\bar{\nu}_{e}$ disappearance in reactor neutrino experiments [9-12]. Using the reactor measurement of $\theta_{13}$, the $\nu_{\mu} \rightarrow \nu_{e}$ appearance mode can be used to explore $C P$ violation, which has yet to be observed in the lepton sector. $C P$ violation, as shown in Eq. (1), is governed by the second term and can be as large as $27 \%$ of the first term for the $\mathrm{T} 2 \mathrm{~K}$ experimental setup when using current values of the neutrino oscillation parameters.

T2K experiment.-T2K operates at the J-PARC facility in Tokai, Japan. A muon neutrino beam is produced from the decay of charged pions and kaons generated by $30 \mathrm{GeV}$ protons hitting a graphite target and focused by three magnetic horns. Downstream of the horns is the decay volume, 96 meters in length, followed by the beam dump and muon monitors (MUMON). The neutrino beam illuminates an on-axis detector and off-axis detectors positioned at an angle of $2.5^{\circ}$ relative to the beam direction. The resulting energy spectrum, peaked at $0.6 \mathrm{GeV}$ for the off-axis detectors, reduces the $\nu_{e}$ contamination and the feed-down backgrounds to the $\nu_{e}$ appearance signal from higher energy neutrinos. The near detector complex at 280 meters from the target is used to measure the neutrino beam direction, spectrum, and composition before oscillations and to measure neutrino cross sections. The complex consists of an on-axis detector (INGRID) and a suite of off-axis detectors (ND280) that reside within a 0.2 T magnet [2]. The SuperKamiokande (SK) 50 kt water Cherenkov detector, situated $295 \mathrm{~km}$ away, is used to detect the oscillated neutrinos.

The results presented here are based on data taken from January 2010 to May 2013. During this period, the proton beam power has steadily increased and reached $220 \mathrm{~kW}$ continuous operation with a world record of $1.2 \times 10^{14}$ protons per pulse. The total neutrino beam exposure at SK corresponds to $6.57 \times 10^{20}$ POT.

Neutrino beam flux.-The neutrino beam flux [13] is predicted by modeling interactions of the primary beam protons in a graphite target using external hadron production data from the CERN NA61/SHINE experiment [14,15] and the FLUKA2008 package [16,17]. GEANT3 [18] with GCALOR [19] simulates propagation of the secondary or tertiary pions and kaons, and their decays into neutrinos. The $\nu_{e}$ component (including a small amount of $\bar{\nu}_{e}$ ) in the beam is estimated to be less than $1 \%$ of the flux below $1.5 \mathrm{GeV}$, and constitutes an irreducible background to the $\nu_{e}$ appearance search. This component is generated predominantly by the decay of muons for $E_{\nu}<1 \mathrm{GeV}$ and by kaons for $E_{\nu}>1 \mathrm{GeV}$. 
The neutrino flux uncertainties are dominated by the hadron production uncertainties, with contributions from the neutrino beam direction and the proton beam uncertainties. The neutrino beam direction, monitored indirectly by MUMON on a spill-by-spill basis, and directly by INGRID [20], was found to be well within the required $\pm 1 \mathrm{mrad}$ during the full run period. INGRID also measured the neutrino interaction rate per POT to be stable within $0.7 \%$. The total systematic error for the absolute flux prediction is evaluated to be $10 \%-15 \%$ in the relevant energy range. Furthermore, the uncertainty on the ratio of the flux predictions at the far and the near detectors is less than $2 \%$ around the peak.

Neutrino interaction simulations and cross section parameters.-The NEUT neutrino interaction generator [21] is used to simulate neutrino interactions in the INGRID, ND280, and SK detectors. At interaction energies typical of the $\mathrm{T} 2 \mathrm{~K}$ beam, the dominant charged current (CC) interactions are charged current quasielastic (CCQE) and single resonant pion production. The cross section parametrization can be divided into two categories: parameters common to interactions at both ND280 and SK, and parameters evaluated separately for the two detectors. Parameters in the first category comprise the axial masses for CCQE $\left(M_{A}^{\mathrm{QE}}\right)$ and single resonant pion production $\left(M_{A}^{\mathrm{RES}}\right)$, and normalizations for $\mathrm{CCQE}, \mathrm{CC}$ single pion, and neutral current (NC) $1 \pi^{0}$ interactions. Parameters in the second category are typically related to the interaction target-primarily carbon at ND280 and oxygen at SKand include Fermi momentum, binding energy, and spectral function modeling for the CCQE nuclear model. Also in this category are normalizations for other $\mathrm{CC}$ and $\mathrm{NC}$ cross sections, the $\nu_{e} / \nu_{\mu} \mathrm{CC}$ cross section ratio, pion production parameters, and final state interactions of pions exiting the nucleus. External data sets, primarily from [22-24], are used to determine the initial values and prior uncertainties of the parameters [4].

ND280 measurements, flux, and common cross section fits.The energy spectrum of the neutrino beam and the neutrino cross section parameters are constrained using $\nu_{\mu} \mathrm{CC}$ interactions in ND280. The fine-grained detectors (FGDs) [25] are scintillator trackers that serve as the primary neutrino target, and the momentum and identity of the particles emerging from the interaction are determined by the time projection chambers (TPCs) [26] interleaved with the FGDs. The muon is assumed to be the highestmomentum, negative-curvature track that emerges from the FGD fiducial volume with an energy deposition consistent with a muon in the TPC downstream of the FGD. Tracks found in the TPC upstream of the FGD are used to veto external background events.

The ND280 analysis includes many improvements over the previous $\mathrm{T} 2 \mathrm{~K}$ electron neutrino appearance measurement [4]. Candidate events are now divided into three samples: $\mathrm{CC}-0 \pi$, dominated by $\mathrm{CCQE}$ interactions;
$\mathrm{CC}-1 \pi^{+}$, dominated by $\mathrm{CC}$ resonant pion production; and $\mathrm{CC}$ other. The samples are defined by the number of pions in the observed final state. A $\pi^{+}$can be identified in one of three ways: an FGD + TPC track with positive curvature and a TPC charge deposition consistent with a pion, an FGD-contained track with a charge deposition consistent with a pion, or a delayed energy deposit due to a decay electron from stopped $\pi^{+} \rightarrow \mu^{+}$in the FGD. To tag a $\pi^{-}$, only negative curvature FGD + TPC tracks are used. A $\pi^{0}$ is identified if there exists a track in the TPC with a charge deposition consistent with an electron from a $\gamma$ conversion. Events containing no pions are classified as $\mathrm{CC}-0 \pi$, events with exactly one $\pi^{+}$and no $\pi^{-}$or $\pi^{0}$ are classified as CC- $1 \pi^{+}$, and all other CC events are classified as CC other. There are 17369, 4047, and 4173 data events in the CC- $0 \pi, \mathrm{CC}-1 \pi^{+}$, and CC-other samples, respectively. The ND280 data set used for this analysis corresponds to $5.90 \times 10^{20}$ POT.

The three samples are fit with 25 beam flux parameters at ND280 (11 $E_{\nu_{\mu}}, 5 E_{\bar{\nu}_{\mu}}, 7 E_{\nu_{e}}$, and $2 E_{\bar{\nu}_{e}}$ bins), 21 cross section parameters (5 in common with SK, and 16 used only for ND280), as well as 210 parameters describing the ND280 detector systematics (10 momentum $\times 7$ angle bins for each sample). The dominant detector uncertainties come from events occurring outside the FGD fiducial volume and from pion reinteractions in the detector. The ND280 measurements constrain the SK flux parameters due to the flux covariance derived from beam simulations. The predicted numbers of ND280 events in Monte Carlo (MC) calculations, using the best-fit parameters, are 17352, 4110 , and 4119 for the CC- $0 \pi, \mathrm{CC}-1 \pi^{+}$, and CC-other samples, respectively. A $\chi^{2}$ goodness-of-fit test returns a $p$ value of 0.66 , indicating no disagreement between the data and the prediction using best-fit parameters. Figure 1 shows the muon momentum distribution of the $\mathrm{CC}-0 \pi$ sample, and the improvement in data and $\mathrm{MC}$ calculations agreement when using the best-fit parameters.

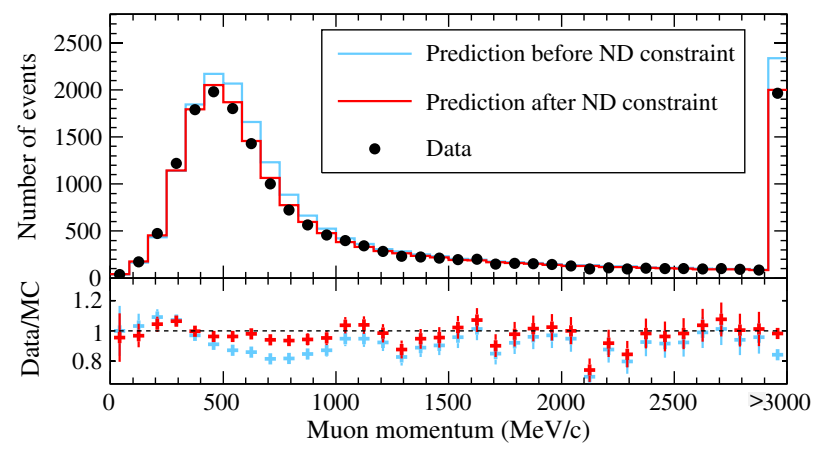

FIG. 1 (color online). The muon momentum distribution for the ND280 CC-0 $\pi$ sample (upper). The black points represent the data, the blue (light gray) histogram shows the MC prediction before data constraint, and the red (dark gray) histogram shows the $\mathrm{MC}$ prediction after constraint. The lower plot shows the ratio of data to $\mathrm{MC}$ for the pre- and post-constraint cases. 
The fit to the ND280 data gives estimates for 22 beam flux parameters at SK, the 5 common cross section parameters, and their covariance. Using the ND280 information reduces the uncertainty on the expected number of electronlike events at SK due to the propagated parameters from $25.9 \%$ to $2.9 \%$.

SK measurements. - The SK detector is composed of an inner detector (ID) and an outer detector (OD). The ID has a water fiducial volume (FV) of $22.5 \mathrm{kt}$ that is equipped with 11129 photomultiplier tubes (PMT) and is surrounded by the $2 \mathrm{~m}$ wide OD. Neutrino events at SK are selected if the Cherenkov ring is consistent with an energy above $30 \mathrm{MeV}$ in the ID with low activity in the OD to reject any entering background or exiting events. These events are labeled fully contained (FC). The FC fiducial volume (FCFV) sample is obtained by applying the further cut that the event vertex is at least $2 \mathrm{~m}$ away from the ID tank wall. A timing cut of -2 to $10 \mu$ s relative to the first beam bunch arrival is applied to distinguish $\mathrm{T} 2 \mathrm{~K}$ data from other neutrino samples such as atmospheric neutrino interactions. The timing cut reduces the contamination from other neutrino sources to 0.0085 events in the full sample.

To select $\nu_{e}$ interaction candidate events in the FCFV sample, a single electronlike Cherenkov ring is required. The reconstructed electron momentum $\left(p_{e}\right)$ is required to exceed $100 \mathrm{MeV} / c$ to eliminate decay electrons from stopping muons generated by $\mathrm{CC}$ interactions and pions in NC interactions. In addition, events are required to have a reconstructed neutrino energy $\left(E_{\nu}^{\mathrm{rec}}\right)$ below $1250 \mathrm{MeV}$. Nearly all of the oscillated $\nu_{e}$ signal events are below this value, while most of the intrinsic beam $\nu_{e}$ background events have higher energies. The $E_{\nu}^{\mathrm{rec}}$ is calculated assuming a CCQE interaction as

$$
E_{\nu}^{\mathrm{rec}}=\frac{m_{p}^{2}-\left(m_{n}-E_{b}\right)^{2}-m_{e}^{2}+2\left(m_{n}-E_{b}\right) E_{e}}{2\left(m_{n}-E_{b}-E_{e}+p_{e} \cos \theta_{\mathrm{e}}\right)},
$$

where $m_{n}\left(m_{p}\right)$ is the neutron (proton) mass, $E_{b}$ is the neutron binding energy in oxygen $(27 \mathrm{MeV}), m_{e}$ is the electron mass, $E_{e}$ is its energy, and $\theta_{\mathrm{e}}$ is the angle of the electron direction relative to the beam direction.

The final selection criterion removes additional $\pi^{0}$ background events using a new reconstruction algorithm, based on an extension of the model described in Ref. [27], to determine the kinematics of all final state particles. The new algorithm is a maximum-likelihood fit in which charge and time probability density functions are constructed for every PMT hit for a given particle hypothesis with a set of 7 parameters: the vertex position, the timing, the direction, and the momentum. Multiple-particle fit hypotheses are constructed by summing the charge contributions from each constituent particle. Different neutrino final states are distinguished by comparing the best-fit likelihood resulting from the fit of each hypothesis. To separate $\pi^{0}$ events from $\nu_{e} \mathrm{CC}$ events, both the reconstructed $\pi^{0}$ mass $\left(m_{\pi^{0}}\right)$ and the

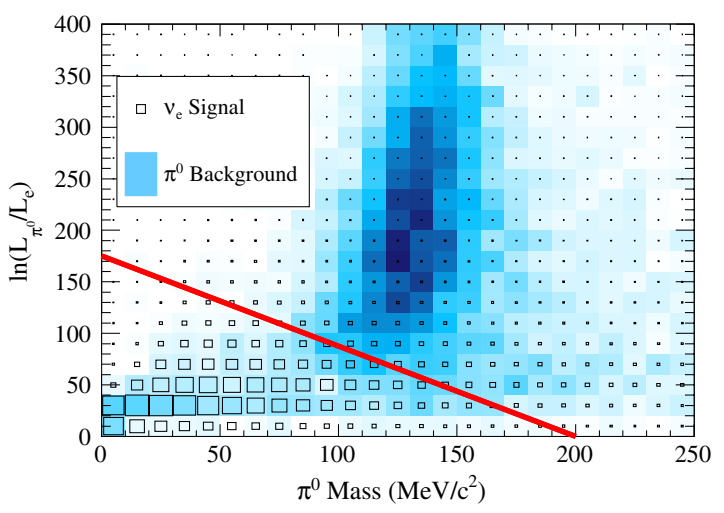

FIG. 2 (color online). The $\ln \left(L_{\pi^{0}} / L_{e}\right)$ vs $m_{\pi^{0}}$ distribution is shown for both signal $\nu_{e}$-CC events (boxes) and background events containing a $\pi^{0}$ (blue scale). The red (gray) line indicates the location of the $\pi^{0}$ rejection cut. Events in the upper right corner are rejected.

ratio of the best-fit likelihoods of the $\pi^{0}$ and electron fits $\left(L_{\pi^{0}} / L_{e}\right)$ are used. Figure 2 shows the $\ln \left(L_{\pi^{0}} / L_{e}\right)$ vs $\pi^{0}$ mass distribution for signal $\nu_{e}-\mathrm{CC}$ events and events containing a $\pi^{0}$ in the MC sample, as well as the rejection cut line. Events that satisfy $\ln \left(L_{\pi^{0}} / L_{e}\right)<175-0.875 \times$ $m_{\pi^{0}}\left(\mathrm{MeV} / c^{2}\right)$ constitute the final $\nu_{e}$ candidate sample. This cut removes $69 \%$ of the $\pi^{0}$ background events relative to the previous $\mathrm{T} 2 \mathrm{~K} \nu_{e}$ appearance selection, with only a $2 \%$ loss in signal efficiency [3].

A summary of the number of events passing each selection cut is shown in Table I. After all cuts, the total number of candidate $\nu_{e}$ events selected in data is 28 , which is significantly larger than the $4.92 \pm 0.55$ expected events for $\theta_{13}=0$. For $\sin ^{2} 2 \theta_{13}=0.1$ and $\delta_{C P}=0$, the expected number is 21.6, as shown in Table I.

The systematic uncertainty due to the SK selection cuts is evaluated using various data and MC samples. The

TABLE I. The expected number of signal and background events passing each selection stage assuming $\sin ^{2} 2 \theta_{13}=0.1$, $\sin ^{2} \theta_{23}=0.5, \quad\left|\Delta m_{32}^{2}\right|=2.4 \times 10^{-3} \mathrm{eV}^{2}, \quad \delta_{C P}=0, \quad$ and $\Delta m_{32}^{2}>0$, compared to the observed number in data. Interactions in the true $\mathrm{FV}$ are based on the $\mathrm{MC}$ truth information while all other numbers are based on the reconstructed information and have been rounded off after addition to avoid rounding error.

\begin{tabular}{|c|c|c|c|c|c|c|}
\hline Selection & Data & $\begin{array}{c}\nu_{\mu} \rightarrow \nu \\
\mathrm{CC}\end{array}$ & $\begin{array}{c}\nu_{\mu}+\bar{\nu}_{\mu} \\
\mathrm{CC}\end{array}$ & $\begin{array}{c}\nu_{e}+\bar{\nu}_{e} \\
\mathrm{CC}\end{array}$ & $\mathrm{NC}$ & $\begin{array}{l}\text { Total } \\
\text { MC }\end{array}$ \\
\hline Interactions in $\mathrm{FV}$ & & 27.1 & 325.7 & 16.0 & 288.1 & 656.8 \\
\hline FCFV & 377 & 26.2 & 247.8 & 15.4 & 83.0 & 372.4 \\
\hline+ Single ring & 193 & 22.7 & 142.4 & 9.8 & 23.5 & 198.4 \\
\hline$+e$-like ring & 60 & 22.4 & 5.6 & 9.7 & 16.3 & 54.2 \\
\hline$+p_{e}>100 \mathrm{MeV} / c$ & 57 & 22.0 & 3.7 & 9.7 & 14.0 & 49.4 \\
\hline +No decay $e$ & 44 & 19.6 & 0.7 & 7.9 & 11.8 & 40.0 \\
\hline$+E_{\nu}^{\mathrm{rec}}<1250 \mathrm{MeV}$ & 39 & 18.8 & 0.2 & 3.7 & 9.0 & 31.7 \\
\hline+ Non- $\pi^{0}$-like & 28 & 17.3 & 0.1 & 3.2 & 1.0 & 21.6 \\
\hline
\end{tabular}


uncertainty for both the FC and the FV selection is 1\%. The decay-electron rejection cut has errors of $0.2 \%-0.4 \%$, depending on neutrino flavor and interaction type. The uncertainties for the single electronlike ring selection and $\pi^{0}$ rejection are estimated by using the SK atmospheric neutrino data and SK cosmic-ray muons. Electron-neutrino CC-enriched control samples based on these cuts were prepared, and the differences between MC predictions and data are used to extract the systematic uncertainty. The uncertainty associated with the $\pi^{0}$ background is determined by constructing a hybrid sample with either an electronlike ring taken from the atmospheric data sample or from decay-electrons selected in the stopping muon data sample, and a MC-generated gamma ray assuming $\pi^{0}$ kinematics. The selection cut systematic uncertainty is calculated to be $1.6 \%$ for signal events and $7.3 \%$ for background events. The total SK selection uncertainty is $2.1 \%$ for the $\nu_{e}$ candidate events assuming $\sin ^{2} 2 \theta_{13}=0.1$.

Additional SK systematic uncertainties are due to finalstate interactions (FSI) of pions that occur inside the target nucleus, as well as secondary interactions (SI) of pions and photonuclear $(\mathrm{PN})$ interactions of photons that occur outside of the target nucleus. The treatment of the FSI and SI uncertainties is the same as in the previous analysis [28]. For this analysis, a new simulation of PN interactions has been added to the SK MC. In the final $\nu_{e}$ event sample, $15 \%$ of the remaining $\pi^{0}$ background is due to events where one of the $\pi^{0}$ decay photons is absorbed in a PN interaction. A systematic uncertainty of $100 \%$ is assumed for the normalization of the PN cross section.

Oscillation analysis.-The neutrino oscillation parameters are evaluated using a binned extended maximum-likelihood fit. The likelihood consists of four components: a normalization term $\left(\mathcal{L}_{\text {norm }}\right)$, a term for the spectrum shape $\left(\mathcal{L}_{\text {shape }}\right)$, a systematics term $\left(\mathcal{L}_{\text {syst }}\right)$, and a constraint term $\left(\mathcal{L}_{\text {const }}\right)$ from other measurements

$$
\begin{aligned}
\mathcal{L}\left(N_{\text {obs }}, \vec{x}, \vec{o}, \vec{f}\right)= & \mathcal{L}_{\text {norm }}\left(N_{\text {obs }} ; \vec{o}, \vec{f}\right) \times \mathcal{L}_{\text {shape }}(\vec{x} ; \vec{o}, \vec{f}) \\
& \times \mathcal{L}_{\text {syst }}(\vec{f}) \times \mathcal{L}_{\text {const }}(\vec{o}),
\end{aligned}
$$

where $N_{\text {obs }}$ is the number of observed events, $\vec{x}$ is a set of kinematic variables, $\vec{o}$ represents oscillation parameters, and $\vec{f}$ describes systematic uncertainties. In the fit, the likelihood is integrated over the nuisance parameters to obtain a marginalized likelihood for the parameters of interest.

$\mathcal{L}_{\text {norm }}$ is calculated from a Poisson distribution using the mean value from the predicted number of $\mathrm{MC}$ events. $\mathcal{L}_{\text {syst }}(\vec{f})$ constrains the 27 systematic parameters from the ND280 fit, the SK-only cross section parameters, and the SK selection efficiencies. Table II shows the uncertainties on the predicted number of signal $\nu_{e}$ events. The $\mathcal{L}_{\text {shape }}$ term uses $x=\left(p_{e}, \theta_{e}\right)$ to distinguish the $\nu_{e}$ signal from backgrounds. An alternative analysis uses $x=E_{\nu}^{\text {rec }}$, the reconstructed neutrino energy. In order to combine the results
TABLE II. The uncertainty ( $\mathrm{rms} /$ mean in $\%)$ on the predicted number of signal $\nu_{e}$ events for each group of systematic uncertainties for $\sin ^{2} 2 \theta_{13}=0.1$ and 0 . The uncorrelated $\nu$ interaction uncertainties are those coming from parts of the neutrino interaction model that cannot be constrained with ND280.

\begin{tabular}{lcc}
\hline \hline Error source [\%] & $\sin ^{2} 2 \theta_{13}=0.1$ & $\sin ^{2} 2 \theta_{13}=0$ \\
\hline Beam flux and near detector & 2.9 & 4.8 \\
(without ND280 constraint) & $(25.9)$ & $(21.7)$ \\
Uncorrelated $\nu$ interaction & 7.5 & 6.8 \\
Far detector and FSI + SI + PN & 3.5 & 7.3 \\
Total & 8.8 & 11.1 \\
\hline \hline
\end{tabular}

presented in this Letter with other measurements to better constrain $\sin ^{2} 2 \theta_{13}$ and $\delta_{C P}$, the $\mathcal{L}_{\text {const }}$ term can also be used to apply additional constraints on $\sin ^{2} 2 \theta_{13}, \sin ^{2} \theta_{23}$, and $\Delta m_{32}^{2}$.

The following oscillation parameters are fixed in the analysis: $\sin ^{2} \theta_{12}=0.306, \Delta m_{21}^{2}=7.6 \times 10^{-5} \mathrm{eV}^{2}$ [29], $\sin ^{2} \theta_{23}=0.5, \quad\left|\Delta m_{32}^{2}\right|=2.4 \times 10^{-3} \mathrm{eV}^{2} \quad[30], \quad$ and $\delta_{C P}=0$. For the normal (inverted) hierarchy case, the best-fit value with a $68 \%$ confidence level (C.L.) is $\sin ^{2} 2 \theta_{13}=0.140_{-0.032}^{+0.038}\left(0.170_{-0.037}^{+0.045}\right)$. Figure 3 shows the best-fit result, with the 28 observed $\nu_{e}$ events. The alternative analysis using $E_{\nu}^{\text {rec }}$ and a profile likelihood method produces consistent best-fit values and nearly identical confidence regions. Figure 4 shows the $E_{\nu}^{\text {rec }}$ distribution with the MC prediction for the best-fit $\theta_{13}$ value in the alternative analysis.

The significance for a nonzero $\theta_{13}$ is calculated to be $7.3 \sigma$, using the difference of $\log$ likelihood values between the best-fit $\theta_{13}$ value and $\theta_{13}=0$. An alternative method of calculating the significance, by generating a large number of toy MC experiments assuming $\theta_{13}=0$, also returns a

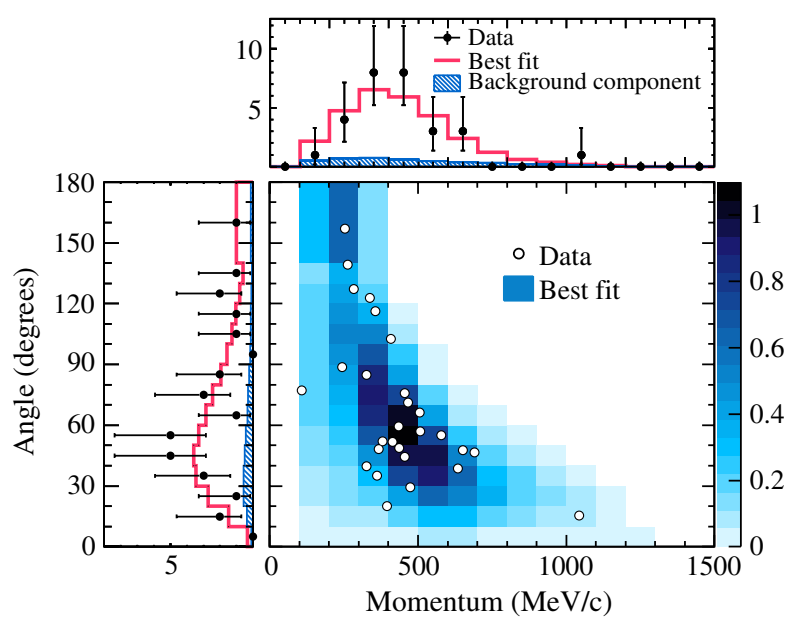

FIG. 3 (color online). The $\left(p_{e}, \theta_{e}\right)$ distribution for $\nu_{e}$ candidate events with the MC prediction using the primary method best-fit value of $\sin ^{2} 2 \theta_{13}=0.140$ (normal hierarchy). 


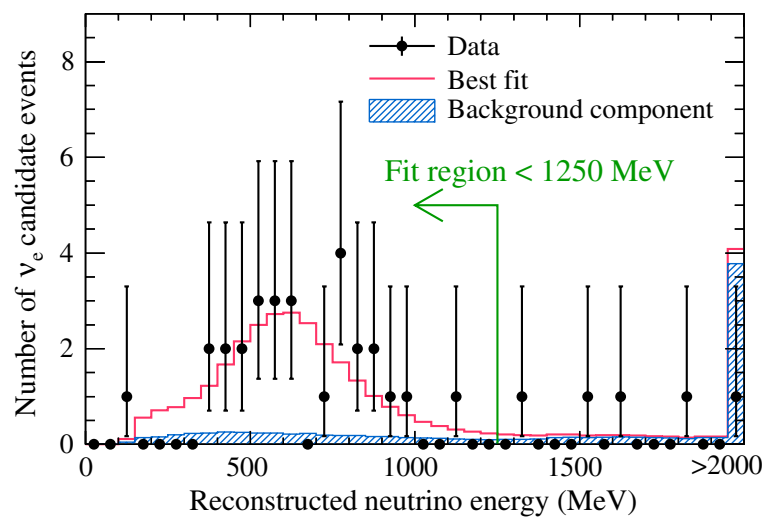

FIG. 4 (color online). The $E_{\nu}^{\text {rec }}$ distribution for $\nu_{e}$ candidate events with the MC prediction at the best fit of $\sin ^{2} 2 \theta_{13}=0.144$ (normal hierarchy) by the alternative binned $E_{\nu}^{\text {rec }}$ analysis.

value of $7.3 \sigma$. These significances were calculated using a test statistic having fixed values for $\theta_{23}$ and $\delta_{C P}$. For any values for these parameters, consistent with their present uncertainties, the significance remains above $7 \sigma$.

As the precision of this measurement increases, the uncertainty from other oscillation parameters becomes increasingly important. The uncertainties on $\theta_{23}$ and $\Delta m_{32}^{2}$ are taken into account in the fit by adding a $\mathcal{L}_{\text {const }}$ term and marginalizing the likelihood over $\theta_{23}$ and $\Delta m_{32}^{2}$. The $\mathcal{L}_{\text {const }}$ term is the likelihood as a function of $\sin ^{2} \theta_{23}$ and $\Delta m_{32}^{2}$, obtained from the $\mathrm{T} 2 \mathrm{~K} \nu_{\mu}$ disappearance measurement [30]. The value of $\delta_{C P}$ and the hierarchy are held fixed in the fit. Performing the fit for all values of $\delta_{C P}$, the allowed $68 \%$ and $90 \%$ C.L. regions for $\sin ^{2} 2 \theta_{13}$ are obtained as shown in Fig. 5. For $\delta_{C P}=0$ and normal (inverted) hierarchy case, the best-fit value with a $68 \%$ C.L. is $\sin ^{2} 2 \theta_{13}=0.136_{-0.033}^{+0.044}\left(0.166_{-0.042}^{+0.051}\right)$. With the current statistics, the correlation between the $\nu_{\mu}$ disappearance and $\nu_{e}$ appearance measurements in $\mathrm{T} 2 \mathrm{~K}$ is negligibly small.

Constraints on $\delta_{C P}$ are obtained by combining our results with the $\theta_{13}$ value measured by reactor experiments. The additional likelihood constraint term on $\sin ^{2} 2 \theta_{13}$ is defined as $\exp \left\{-\left(\sin ^{2} 2 \theta_{13}-0.098\right)^{2} /\left[2\left(0.013^{2}\right)\right]\right\}$, where 0.098 and 0.013 are the averaged value and the error of $\sin ^{2} 2 \theta_{13}$ from PDG2012 [9]. The $-2 \Delta \ln \mathcal{L}$ curve as a function of $\delta_{C P}$ is shown in Fig. 6, where the likelihood is marginalized over $\sin ^{2} 2 \theta_{13}, \sin ^{2} \theta_{23}$, and $\Delta m_{32}^{2}$. The combined T2K and reactor measurements prefer $\delta_{C P}=-\pi / 2$. The $90 \%$ C.L. limits shown in Fig. 6 are evaluated by using the FeldmanCousins method [31] in order to extract the excluded region. The data exclude $\delta_{C P}$ between $0.19 \pi$ and $0.80 \pi$ $(-\pi$ and $-0.97 \pi$, and $-0.04 \pi$ and $\pi)$ with normal (inverted) hierarchy at $90 \%$ C.L.

The maximum value of $-2 \Delta \ln \mathcal{L}$ is 3.38 (5.76) at $\delta_{C P}=\pi / 2$ for the normal (inverted) hierarchy case. This value is compared with a large number of toy MC experiments, generated assuming $\delta_{C P}=-\pi / 2, \sin ^{2} 2 \theta_{13}=0.1$,

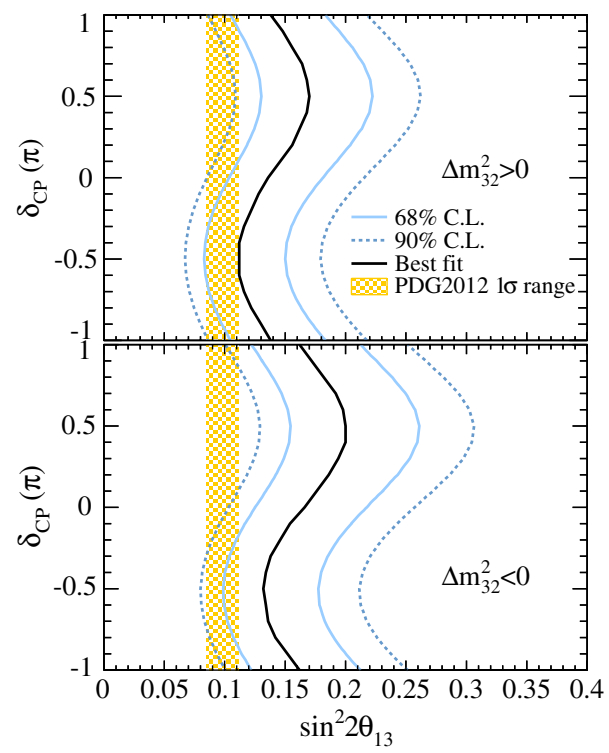

FIG. 5 (color online). The $68 \%$ and $90 \%$ C.L. allowed regions for $\sin ^{2} 2 \theta_{13}$, as a function of $\delta_{C P}$ assuming normal hierarchy (top) and inverted hierarchy (bottom). The solid line represents the best fit $\sin ^{2} 2 \theta_{13}$ value for given $\delta_{C P}$ values. The values of $\sin ^{2} \theta_{23}$ and $\Delta m_{32}^{2}$ are varied in the fit with the constraint from [30]. The shaded region shows the average $\theta_{13}$ value from the PDG2012 [9].

$\sin ^{2} \theta_{23}=0.5$, and $\Delta m_{32}^{2}=2.4 \times 10^{-3} \mathrm{eV}^{2}$. The MC averaged value of $-2 \Delta \ln \mathcal{L}$ at $\delta_{C P}=\pi / 2$ is 2.20 (4.10) for the normal (inverted) hierarchy case, and the probability of obtaining a value greater or equal to the observed value is $34.1 \%(33.4 \%)$. With the same MC settings, the expected $90 \%$ C.L. exclusion region is evaluated to be between $0.35 \pi$ and $0.63 \pi(0.09 \pi$ and $0.90 \pi)$ radians for the normal (inverted) hierarchy case.

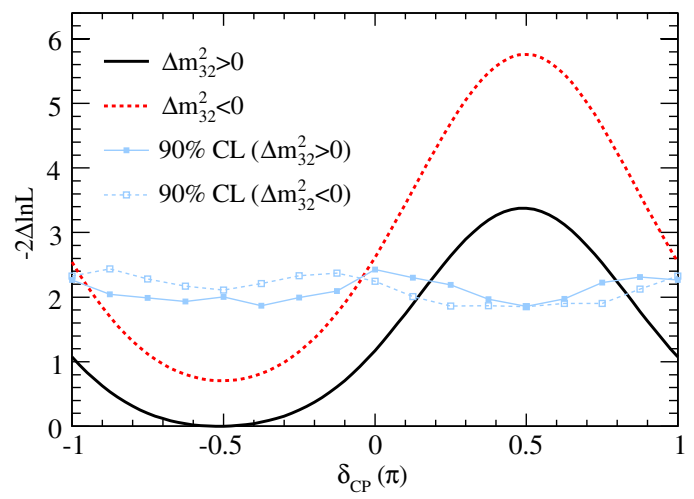

FIG. 6 (color online). The $-2 \Delta \ln \mathcal{L}$ value as a function of $\delta_{C P}$ for normal hierarchy (solid line) and inverted hierarchy (dotted line). The likelihood is marginalized over $\sin ^{2} 2 \theta_{13}, \sin ^{2} \theta_{23}$, and $\Delta m_{32}^{2}$. The solid (dotted) line with markers corresponds to the 90\% C.L. limits for normal (inverted) hierarchy, evaluated by using the Feldman-Cousins method. The $\delta_{C P}$ regions with values above the lines are excluded at $90 \%$ C.L. 
Conclusions.-T2K has made the first observation of electron neutrino appearance in a muon neutrino beam with a peak energy of $0.6 \mathrm{GeV}$ and a baseline of $295 \mathrm{~km}$. With the fixed parameters $\left|\Delta m_{32}^{2}\right|=2.4 \times 10^{-3} \mathrm{eV}^{2}$, $\sin ^{2} \theta_{23}=0.5, \delta_{C P}=0$, and $\Delta m_{32}^{2}>0\left(\Delta m_{32}^{2}<0\right)$, a best-fit value of $\sin ^{2} 2 \theta_{13}=0.140_{-0.032}^{+0.038}\left(0.170_{-0.037}^{+0.045}\right)$ is obtained, with a significance of $7.3 \sigma$ over the hypothesis of $\sin ^{2} 2 \theta_{13}=0$. When combining the $\mathrm{T} 2 \mathrm{~K}$ result with the world average value of $\theta_{13}$ from reactor experiments, some values of $\delta_{C P}$ are disfavored at the $90 \%$ C.L.

$\mathrm{T} 2 \mathrm{~K}$ will continue to take data to measure the neutrino oscillation parameters more precisely and to further explore $C P$ violation in the lepton sector.

We thank the J-PARC staff for superb accelerator performance and the CERN NA61 collaboration for providing valuable particle production data. We acknowledge the support of MEXT, Japan; NSERC, NRC, and CFI, Canada; CEA and CNRS/IN2P3, France; DFG, Germany; INFN, Italy; Ministry of Science and Higher Education, Poland; RAS, RFBR, and MES, Russia; MICINN and CPAN, Spain; SNSF and SER, Switzerland; STFC, UK; and DOE, USA We also thank CERN for the UA1/ NOMAD magnet, DESY for the HERA-B magnet mover system, NII for SINET4, the WestGrid and SciNet consortia in Compute Canada, and GridPP, UK. In addition participation of individual researchers and institutions has been further supported by funds from: ERC (FP7), EU; JSPS, Japan; Royal Society, UK; DOE Early Career program, USA.

\footnotetext{
*Deceased.

${ }^{\dagger}$ Also at J-PARC, Tokai, Japan.

*Also at Institute of Particle Physics, Canada.

${ }^{\S}$ Also at Kavli IPMU (WPI), the University of Tokyo, Japan.

"Also at Moscow Institute of Physics and Technology and National Research Nuclear University (MEPhI), Moscow, Russia.

"Also at JINR, Dubna, Russia.

** Also at BMCC/CUNY, Science Department, New York, New York, USA.
}

[1] Y. Fukuda et al. (Super-Kamiokande Collaboration) Phys. Rev. Lett. 81, 1562 (1998).

[2] K. Abe et al. (T2K Collaboration), Nucl. Instrum. Methods Phys. Res., Sect. A 659, 106 (2011).

[3] K. Abe et al. (T2K Collaboration) Phys. Rev. Lett. 107, 041801 (2011).

[4] K. Abe et al. (T2K Collaboration) Phys. Rev. D 88, 032002 (2013).
[5] Z. Maki, M. Nakagawa, and S. Sakata, Prog. Theor. Phys. 28, 870 (1962).

[6] B. Pontecorvo, Sov. Phys. JETP 26, 984 (1968).

[7] M. Freund, Phys. Rev. D 64, 053003 (2001).

[8] P. Adamson et al. (MINOS Collaboration), Phys. Rev. Lett. 110, 171801 (2013).

[9] J. Beringer et al. (Particle Data Group), Phys. Rev. D 86, 010001 (2012).

[10] F. An et al. (Daya Bay Collaboration) Phys. Rev. Lett. 108, 171803 (2012).

[11] J. Ahn et al. (RENO Collaboration), Phys. Rev. Lett. 108, 191802 (2012).

[12] Y. Abe et al. (Double Chooz Collaboration), Phys. Rev. Lett. 108, 131801 (2012).

[13] K. Abe et al., (T2K Collaboration), Phys. Rev. D 87, 012001 (2013).

[14] N. Abgrall et al. (NA61/SHINE Collaboration), Phys. Rev. C 84, 034604 (2011).

[15] N. Abgrall et al. (NA61/SHINE Collaboration), Phys. Rev. C 85, 035210 (2012).

[16] A. Ferrari, P. R. Sala, A. Fasso, and J. Ranft, Report No. CERN-2005-010.

[17] G. Battistoni, S. Muraro, P. R. Sala, F. Cerutti, A. Ferrari, et al., AIP Conf. Proc. 896, 31 (2007).

[18] R. Brun, F. Carminati, and S. Giani, Report No. CERNW5013 (1994).

[19] C. Zeitnitz and T. Gabriel, in Proceedings of the Third International Conference on Calorimetry in High Energy Physics, Corpus Christi, Texas, 1992, edited by Phyllis Hale and James Siegrist (World Scientific, Singapore, 1993).

[20] K. Abe et al. (T2K Collaboration), Nucl. Instrum. Methods Phys. Res., Sect. A 694, 211 (2012).

[21] Y. Hayato, Acta Phys. Pol. B 40, 2477 (2009).

[22] A. A. Aguilar-Arevalo et al. (MiniBooNE Collaboration), Phys. Rev. D 83, 052009 (2011).

[23] A. A. Aguilar-Arevalo et al. (MiniBooNE Collaboration), Phys. Rev. D 83, 052007 (2011).

[24] A. A. Aguilar-Arevalo et al. (MiniBooNE Collaboration), Phys. Rev. D 81, 013005 (2010).

[25] P. Amaudruz et al. (T2K ND280 FGD Group), Nucl. Instrum. Methods Phys. Res., Sect. A 696, 1 (2012).

[26] N. Abgrall et al. (T2K ND280 TPC Group), Nucl. Instrum. Methods Phys. Res., Sect. A 637, 25 (2011).

[27] R. B. Patterson, E. M. Laird, Y. Liu, P. D. Meyers, I. Stancu, and H. A. Tanaka, Nucl. Instrum. Methods Phys. Res., Sect. A 608, 206 (2009).

[28] K. Abe et al. (T2K Collaboration), Phys. Rev. D 88, 032002 (2013).

[29] G. L. Fogli, E. Lisi, A. Marrone, A. Palazzo, and A. M. Rotunno, Phys. Rev. D 84, 053007 (2011).

[30] K. Abe et al. (T2K Collaboration), Phys. Rev. Lett. 111, 211803 (2013).

[31] G. J. Feldman and R. D. Cousins, Phys. Rev. D 57, 3873 (1998). 